\title{
AVALIAÇÃO DO INOCULANTE “GRAMINANTE” NA CULTURA DE MILHO
}

\author{
EVALUATION OF INOCULANT “GRAMINANTE” IN CORN CROP
}

\author{
Ben-Hur Costa de Campos ${ }^{1}$ Sergiomar Theisen ${ }^{2}$ Valderi Gnatta ${ }^{3}$
}

- NOTA -

RESUMO

\begin{abstract}
Com o objetivo de avaliar o inoculante "Graminante", foram realizados, na safra 1996/97, dois experimentos com a cultura de milho, em área experimental localizada na FUNDACEP FECOTRIGO, Cruz Alta, RS, conduzidos no sistema plantio direto. O produto comercial foi testado individualmente e associado à adubação nitrogenada. Os tratamentos foram: l) testemunha sem inoculação e sem fertilizante nitrogenado (N);2) com "Graminante"; 3) com N na semeadura e em cobertura: 4) com "Graminante" + N na semeadura e em cobertura; 5) com "Graminante" $+N$ na semeadura e, 6) com "Graminante" $+N$ em cobertura. Foram avaliados o número de plantas e de espigas, a estatura de plantas e o rendimento de grãos. O produto comercial "Graminante " não apresentou resposta na cultura de milho.
\end{abstract}

Palavras-chave: fixação biológica de Ni, inoculação, AwspiriUum, milho.

\section{SUMMARY}

To evaluate the inoculant "Graminante", two field experiments were carried, in the 1996/97 cropping season, with com crop, out at FUNDACEP FECOTRIGO, in Cruz Alta, RS, Brazil. The commerciat product was tested isolated and in association with nitrogen fertilization. The treatments were as follows: l) Check without inoculation and without nitrogen fertilizer (N): 2) "Graminante" inoculam; 3) Nitrogen fertilizer apptied at the planting date and broadcasting. ; 4) "Graminante" imculant $+N$ fertilizer ai planting date and broadcasting. 5) "Graminante" inoculam $+N$ fertilizer at planting date; 6)
"Graminante" inoculam $+N$ fertilizer broadcasting. The parameters evaluated were: number ofplants and ofspikes, plantheight and grain yield. The inoculam "Graminante" studied did not show influencefor the com crop.

Key words: nitrogen biotogical fixation, inoculation, Awspirilium, corn.

O grande interesse na fixação biológica em gramíneas é devido à maior facilidade de aproveitamento de água das mesmas em relação às leguminosas, pela maior efetividade fotossintética. As gramíneas apresentam um sistema radicular fasciculado, tendo vantagens sobre o sistema pivotante das leguminosas para extrair água e nutrientes do solo; e por serem as gramíneas largamente utilizadas como alimento pelo homem. Por isso, mesmo que apenas uma parte do $\mathrm{N}$ pudesse ser fornecida pela associação com bactérias fixadoras, a economia em adubos nitrogenados seria igual ou superior àquela verificada com as leguminosas que podem ser auto-suficientes em nitrogénio (DÕBEREINER, 1992).

Bactérias do género Azospirilium são microrganismos fixadores de nitrogénio atmosférico em vida livre, também chamados diazotrófícos. Essas bactérias, em regiões tropicais e subtropicais, ocorrem em números entre 10 a 10 por grama de

\footnotetext{
${ }^{1}$ Engenheiro Agrônomo, MSc., Pesquisador da Fundação Centro de Experimentação e Pesquisa Fecotrigo (FUNDACEP FECOTRIGO), RS 342, km 14, CP 10, 98100-970. Cruz Alta - RS. E-mail: fundacep@azcomnet.com.br. Autor para correspondência.

${ }^{2}$ Aluno do Curso de Agronomia, Universidade de Cruz Alta, Cruz Alta - RS. Bolsista do CIEE.

${ }^{3}$ Técnico Agrícola, FUNDACEP FECOTRIGO.
} 
solo, e em números ainda maiores na superfície de raízes de cereais e gramíneas forrageiras (DÕBEREINER et al., 1990).

A contribuição em fixação biológica de N2 por bactérias assimbióticas é muito restrita, devido a grande necessidade de material energético requerida (RUSCHEL \& PONTES, 1992). O principal efeito dessas bactérias está, principalmente, na promoção do crescimento radicular das plantas, pela produção de substâncias promotoras de crescimento (OKON \& LABANDERAGONZALEZ, 1994;

ALVAREZ et al., 1996), sendo que a resposta da inoculação de cereais com essas bactérias depende do estabelecimento de estirpes selecionadas sob condições de campo (DÕBEREINER $\boldsymbol{e t}$ al., 1990). Esse estabelecimento torna-se difícil em solos de regiões tropicais e subtropicais, nos quais a incidência natural de Azospírilium é relativamente grande e não há especificidade nas associações de gramíneas com essa bactéria (DÕBEREINER $\boldsymbol{e t}$ al., 1990).

Recentemente, têm sido introduzidos no Brasil inoculantes contendo cepas de Azospirilium, com o nome comercial "Graminante", havendo, contudo, carência de informações relativas ao seu potencial agronómico e retomo económico para o agricultor. Dessa forma, o objetivo deste trabalho foi testar a eficiência agronómica do produto comercial "Graminante" na cultura de milho.

$\mathrm{Na}$ safra 1996/97, foram conduzidos dois experimentos com a cultura do milho, sendo que um experimento foi em resteva de ervilhaca e o outro em resteva de trigo, instalados a campo, em áreas experimentais da FUNDACEP FECOTRIGO, Cruz Alta, RS.

O solo das áreas experimentais é classificado como latossolo vermelho-escuro, fase argilosa, pertencente à unidade de mapeamento Passo Fundo, com as seguintes características na profundidade de $\mathrm{O}$ a $20 \mathrm{~cm}$, na área de resteva de ervilhaca: argila $480 \mathrm{~g} \mathrm{~kg}^{-1}$; $\mathrm{pH}$ (água) 5,$3 ; \mathrm{P} 22,7 \mathrm{mg}$

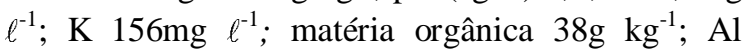
0,0 cmolc $\ell^{-1}$; Ca $6,6 \mathrm{cmolc} \ell^{-1}$; Mg 2,7 cmolc $\ell^{-1}$; CTC $14,0 \mathrm{cmolc} \ell^{-1}$; H+A1 4,3cmolc $\ell^{-1}$. Na área de resteva de trigo, as características, na mesma profundidade, foram: argila $390 \mathrm{~g} \mathrm{~kg}^{-1} ; \mathrm{pH}$ (água) 6,$2 ; \mathrm{P} 31,8 \mathrm{mg} \ell^{-1}$; K264mg $\ell^{-1}$; matéria orgânica $45 \mathrm{~g}$ $\mathrm{kg}^{-1} ; \mathrm{Al} 0,0 \mathrm{cmol}_{\mathrm{c}} \ell^{-1} ; \mathrm{Ca} 10.1 \mathrm{cmol}_{\mathrm{c}} \ell^{-1} ; \mathrm{Mg} 5,0 \mathrm{cmol}_{\mathrm{c}}$ $\ell^{-1}$; CTC $17,8 \mathrm{cmol}_{\mathrm{c}} \ell^{-1} ; \mathrm{H}+\mathrm{A} 12, \mathrm{lcmol}_{\mathrm{c}} \ell^{-1}$.

O produto comercial "Graminante", para a cultura do milho, foi fornecido pela Empresa Laboratórios Alquimia SÁ, com sede na Argentina. Segundo o fabricante, trata-se de um inoculante, composto por bactérias do género Azospirilium e tendo como veículo carbonato de cálcio e carbonato de magnésio. A aplicação do produto foi feita a seco, conforme recomendação do fabricante, e realizada em laboratório imediatamente antes da semeadura. Foi utilizada a dose de $200 \mathrm{~g}$ do produto comercial por $50 \mathrm{~kg}$ de sementes. $\mathrm{O}$ fabricante não especifica $\mathrm{o}$ número de bactérias por grama de inoculante.

A semeadura foi realizada mecanicamente no sistema plantio direto, com espaçamento de 0,80m entre linhas, iniciando-se pêlos tratamentos que não continham inoculante, para diminuir os riscos de contaminação. Ela foi realizada em 16/10/96 na resteva de ervilhaca e, em 14/11/96 na resteva de trigo, com o híbrido Pionner 3069. As parcelas tiveram $3,0 \times 5,0 \mathrm{~m}$, com bordaduras de $0,5 \mathrm{~m}$ nas extremidades e uma linha da cultura em cada lateral. Entre as parcelas, ruas com 1,0m e entre os blocos, as ruas foram de $6,0 \mathrm{~m}$. O delineamento foi de blocos ao acaso com cinco repetições. A adubação foi realizada com $60 \mathrm{~kg} / \mathrm{ha}$ de $\mathrm{P}_{2} \mathrm{O}_{5}$ e 90 $\mathrm{kg} / \mathrm{há}^{-1}$ de $\mathrm{K}_{2} \mathrm{O}$, à lanço. Os tratamentos foram: 1) testemunha sem inoculação e sem fertilizante nitrogenado (N); 2) com "Graminante"; 3) com N na semeadura e em cobertura; 4) com "Graminante" + $\mathrm{N}$ na semeadura e em cobertura; 5) com "Graminante" + N na semeadura e, 6) com "Graminante" + N em cobertura. Na adubação nitrogenada de semeadura, foi aplicado $20 \mathrm{~kg}$ de $\mathrm{N} / \mathrm{ha}$ e, na adubação de cobertura, $80 \mathrm{~kg}$ de N/ha, realizada com as plantas entre 40 a $60 \mathrm{~cm}$ de estatura. Foram avaliados o número de plantas, de espigas, a estatura de plantas e o rendimento de grãos a $13 \%$ de umidade. Para a estatura de plantas foi feita a média de 5 plantas por parcela. Os resultados foram avaliados pela análise da variância e as médias dos tratamentos foram comparadas pelo teste de Duncan em nível de $5 \%$.

Não houve diferenças estatísticas entre os tratamentos para número de plantas, de espigas e a estatura de plantas, nas duas áreas avaliadas (Tabela 1).

Para rendimento de grãos, o experimento instalado sobre resteva de ervilhaca não apresentou diferenças estatísticas entre os tratamentos. Isso pode ser atribuído ao efeito da resteva da leguminosa, que provavelmente minimizou as diferenças de nitrogénio entre os tratamentos, fornecendo esse nutriente através da decomposição de seus resíduos, com baixa relação carbono/nitrogénio. Na resteva de trigo, houve diferenças entre os tratamentos, destacando-se aquelas onde foi aplicado no milho fertilizante nitrogenado, principalmente nas maiores doses. Com relação ao Graminante, este não apresentou resposta para rendimento de grãos, pois os tratamentos com 
dose equivalente de $\mathrm{N}$ mineral, com e sem o produto, não apresentaram diferenças entre si. Esses resultados concordam com CAMPOS et al. (1999) que, trabalhando com o inoculante Graminante para as culturas de trigo e aveia, também não encontraram respostas agronómicas favoráveis ao produto em nenhum dos parâmetros analisados.
CAMPOS, B.C. de, THEISEN, S., GNATTA, V. Avaliação do inoculante "Graminante" nas culturas de trigo e aveia. Ciência Rural, Santa Maria, v.29, n.3, p.401-407, 1999.

DÕBEREINER, J. Fixação de nitrogénio em associação com gramíneas. In.: CARDOSO, E.J.B.N., TSAI, S.M., NEVES, M.C.P. Microbiologia do solo. Campinas : SBCS, 1992.

\section{Tabela 1 - Número de plantas, número de espigas, estatura de plantas e rendimento de grãos de milho, em área sob resteva de ervilhaca e área sob resteva de trigo, com a aplicação do produto comercial "Graminante". Média de 5 repetições. FUNDACEP FECOTRIGO, 1996/97. Cruz Alta, RS.}

\begin{tabular}{|c|c|c|c|c|}
\hline Tratamento & $\begin{array}{c}\text { Número de } \\
\text { plantas }\end{array}$ & $\begin{array}{c}\text { Número de } \\
\text { espigas }\end{array}$ & $\begin{array}{c}\text { Estatura de } \\
\text { plantas }(\mathrm{cm})\end{array}$ & $\begin{array}{l}\text { Rendimento de } \\
\text { grãos (kg/ha) }\end{array}$ \\
\hline \multicolumn{5}{|l|}{ Área de ervilhaca } \\
\hline 1) Testemunha & $41 \mathrm{~ns}^{(2)}$ & $36 \mathrm{~ns}$ & $1,77 \mathrm{~ns}$ & $6.325 \mathrm{~ns}$ \\
\hline 2) Graminante & 38 & 34 & 1,80 & 6.202 \\
\hline 3) Fertilizante $N$ (semeadura + cobertura) ${ }^{(1)}$ & 41 & 38 & 1,87 & 6.813 \\
\hline 4) Graminante + fertilizante $N$ (semeadura + cobertura) & 40 & 38 & 1,83 & 7.098 \\
\hline 5) Graminante + fertilizante $N$ (semeadura) & 38 & 35 & 1,86 & 6.972 \\
\hline 6) Graminante + fertilizante $N$ (cobertura) & 36 & 34 & 1,84 & 7.061 \\
\hline $\mathrm{CV}(\%)$ & 10,7 & 10,6 & 4,8 & 11,1 \\
\hline \multicolumn{5}{|l|}{ Área de trigo } \\
\hline 1) Testemunha & $32 \mathrm{~ns}$ & $30 \mathrm{~ns}$ & $1,79 \mathrm{~ns}$ & $5.484 \mathrm{c}$ \\
\hline 2) Graminante & 30 & 28 & 1,79 & $5.121 \mathrm{c}$ \\
\hline 3) Fertilizante $N$ (semeadura + cobertura) & 32 & 34 & 1,87 & $7.702 \mathrm{a}$ \\
\hline 4) Graminante + fertilizante $N$ (semeadura + cobertura) & 24 & 26 & 1,84 & $7.427 \mathrm{ab}$ \\
\hline 5) Graminante + fertilizante $N$ (semeadura) & 29 & 28 & 1,85 & $6.665 \mathrm{~b}$ \\
\hline 6) Graminante + fertilizante $N$ (cobertura) & 28 & 28 & 1,85 & $7.512 \mathrm{ab}$ \\
\hline $\mathrm{CV}(\%)$ & 15,7 & 14,1 & 3,2 & 9,9 \\
\hline
\end{tabular}

(1) semeadura: $20 \mathrm{~kg}$ de N/ha; cobertura: $80 \mathrm{~kg}$ de N/ha.

(2) $\mathrm{ns}=$ não significativo pelo teste $\mathrm{F}$ a $5 \%$ de probabilidade.

É preciso testar as estirpes e buscar aquelas melhor adaptadas a cada região, em termos de clima, sistema de manejo e cultivares (DÕBEREINER \& PEDROSA, 1987; MARTIN \& DIDONET, 1996; SAUBIDET \& BARNIEX, 1996) e, posteriormente, introduzi-las no produto comercial Graminante, pois a estirpe presente, além de não especificada pelo fabricante, não foi suficientemente eficiente. Uma vantagem verificada no produto é a sua formulação. $\mathrm{O}$ veículo a seco facilita a aplicação do produto na semente, se associado a estirpes eficientes, certamente, permitirá uma maior aceitação por parte do agricultor (CAMPOS et al., 1999).

\section{REFERÊNCIAS BIBLIOGRÁFICAS}

ALVAREZ, M.L, SUELDO, RJ., BARASSI, C.A. Effect of Awspirilium on coleoptile growth in wheat seediings under water stress. Cereal Research Communications, Szeged, v.24,n.1,p.101-107,1996. p.173-180.

DÕBEREINER, J., PEDROSA, F.O. in nonleguminous crop plants. Nitrogen-fixing bactéria Madison : Science Tech, 1987. 155 p.

DÕBEREINER, J.; PAULA, M.A. de, MONTEIRO, E.M.S. A pesquisa em microbiologia do solo no Brasil. Revista Brasileira de Biologia, Rio de Janeiro, v.50, p.841-854,1990.

MARTIN, C.C.G., DIDONET, A.D. Acumulación de nitrito y N20 en aislados de Awspirilium sp. de raíces de trigo (Triticum aestivum). In: REUNIÓN LATINOAMERICANA DE RHIZOBIOLOGÍA, 28, Santa Cruz de Ia Sierra, 1996. Memórias... Santa Cruz de Ia Sierra : Asociación Latinoamericana de Rhizobiología, 1996. 547p. p.291-292.

OKON, Y., LABANDERA-GONZALEZ, C.A. Agronomic appiications of Awspirilium; an evaluation of 20 years woridwide field inoculation. Soil Biology and Biochemistry, Oxford, v.26, p. 1591-1 601,1994.

RUSCHEL, A.P., PONTES, M.C.F. Fixação biológica de nitrogénio por microrganismos assimbióticos. In.: 
CARDOSO, E.J.B.N., TSAI, S.M., NEVES, M.C.P Microbiologia dosolo. Campinas : SBCS, 1992. p. 181-200.

SAUBIDET, M.L, BARNIEX, A.J. Crecimiento y fijación de nitrógeno en plantas de trigo con Azospirilium sp. m: REUNIÓN LATINOAMERICANA DE RHIZOBIOLOGÍA, 28, Santa Cruz de Ia Sierra, 1996. Memórias... Santa Cruz de Ia Sierra : Asociación Latinoamericana de Rhizobiología, 1996. 547p. p.533-535.

Ciência Rural, v. 30, n. 4, 2000. 